\title{
"Normalisierung nach rechts": Politischer Diskurs im Spannungsfeld von Neoliberalismus, Populismus und kritischer Öffentlichkeit
}

\author{
Ruth Wodak (Lancaster)
}

\begin{abstract}
In recent years, we can observe a shift towards the right, in politics and the related political discourse. This paper analyses this development for debates on migration in Austria, while drawing on the concept of "normalisation". The basic assumption is illustrated with an example of Austrian debates following the terrorist attacks in Paris, 7.-9.01.2015. In the quantitative and qualitative analysis of a complete sample of 72 newspaper articles in the period of two weeks in January/February 2015, it becomes obvious that the notion of "unwillingness to integrate" (Integrationsunwilligkeit), a completely vague notion (a "floating signifier") which remains undefined and was used only by the right-wing populist party FPÖ in the 1990s, has since moved to the middle of the political spectrum and was suddenly employed by the political mainstream in 2014 and 2015. Moreover, the analysis provides some evidence for the merging of two completely unrelated arguments: an argument about how to deal with noncompliance of adolescent migrant school children with an argument about the prevention of radicalisation in society.
\end{abstract}

\section{$1 \quad$ Newspeak, Normalisierung und Floating Signifiers}

Im April 1946, noch unter dem Eindruck des Zweiten Weltkrieges und der allseits gegenwärtigen Propaganda der beteiligten Nationalstaaten verfasste der bekannte britische Schriftsteller und Essayist George Orwell einen kurzen Aufsatz, der inzwischen zur Pflichtlektüre aller SprachwissenschaftlerInnen geworden ist, die sich mit der Analyse politischer Kommunikation befassen: "Politics and the English Language". Vieles, was Orwell in seinem Aufsatz und auch in den nachfolgenden Werken, wie "1984" oder "Animal Farm" damals als Fiktion beschreibt, ist heute Realität geworden. Eine berühmt gewordene Passage möge als Einstimmung in die folgenden Ausführungen dienen:

In our time, political speech and writing are largely the defence of the indefensible. Thus political language has to consist largely of euphemism, question-begging and sheer cloudy vagueness. [...] When the general atmosphere is bad, language must suffer. [...]. Phrases like a not unjustifiable assumption, leaves much to be desired, would serve no good purpose, a consideration which we should do well to bear in mind, are a continuous temptation, a packet of aspirins always at one's elbow. This invasion of one's mind by ready-made phrases (lay the foundations, 
achieve a radical transformation) can only be prevented if one is constantly on guard against them, and every such phrase anaesthetizes a portion of one's brain.

(Orwell 1946: 431)

Orwell befasste sich in seinen Büchern vor allem mit der Sprache totalitärer Regime. Viele linguistische Verfahren, wie der Gebrauch von Euphemismen, seltener wie auch fossilisierter Metaphern, des Passivs, das zur Tilgung von Akteuren dient, und prätentiöser Fremdworte finden sich auch heute in der Politik, nicht nur in totalitären sondern durchaus auch in demokratischen Systemen.

Im vorliegenden Aufsatz fokussiere ich vor allem die von Orwell monierte intentionale Vagheit und das sogenannte "Doublethink" bei der Analyse rezenter politischer Debatten zur Integration von MigrantInnen in Österreich (vgl. unten). Vagheit bezieht sich auf das In-dieWelt Setzen von schwammigen, wenn nicht sogar bedeutungsleeren Begriffen; Doublethink hingegen weist darauf hin, dass manchmal widersprüchliche Bedeutungen mit einem Begriff verbunden und akzeptiert sein können. In seinem Roman "1984" ist das "Ministry of Truth" mit der Kontrolle und Verbreitung solcher Begriffe befasst, beispielsweise mit der Gleichsetzung von Gegensätzen, wie "WAR IS PEACE" oder "IGNORANCE IS STRENGTH". Die solcherart definierte Sprache heißt "Newspeak".

Der kürzlich verstorbene argentinische Philosoph und Sprachtheoretiker Ernesto Laclau, der Jahrzehnte an der Essex University, UK, lehrte, machte ähnliche Beobachtungen in seinen berühmt gewordenen Schriften zur politischen Theorie, der "Discourse Theory": Demnach sei "'the political' the realm of conflict and contestation; of breaking and making new and old meanings" (Kolvraa, in Druck).

Laclau (und Chantal Mouffe) beschäftigten sich vor allem mit umkämpften Begriffen, mit Momenten der Krise (der sogenannten "dislocation"), wo solche Bedeutungskämpfe offensichtlich werden und wo es darum geht, die Macht über die Begriffsdefinition zu erhalten und damit die jeweils hegemoniale Bedeutung zu definieren - also jene Bedeutung, die in der Öffentlichkeit Geltung besitzen soll und auch durchaus kontroversielle politische Entscheidungen legitimieren soll. Beispiele dazu gibt es natürlich viele, wie etwa die Auseinandersetzungen um Konzepte wie Demokratie oder Freiheit über die Jahrhunderte hinweg. In diesen Auseinandersetzungen geht es darum, bestimmte vage Begriffe, die Laclau als "floating signifiers" bezeichnet, in ein kohärentes Narrativ, eine Ideologie oder Weltanschauung, in ein politisches Programm einzufügen.

A floating signifier is an element who has not (yet) received a definite meaning, either because a process of dislocation has detached it from its traditional sedimented position or because various political forces seek to ascribe it meaning in radically different ways.

(Laclau/Mouffe 1985/2001: 113)

Es geht also um einen Kampf der Bedeutungen wichtiger politischer Begriffe, die jeweils symbolisch für ganze Gedankengebäude, also metonymisch für eine Ideologie, stehen und emotional besetzt sind; dies um eindeutig und überzeugend zu wirken, damit sie von vielen Menschen aufgegriffen und weiter verwendet werden.

Ein weiteres theoretisches Konzept muss in diesem Zusammenhang noch kurz vorgestellt werden: Der Literaturwissenschaftler Jürgen Link (2014) beschäftigt sich seit vielen Jahren 
eingehend mit den Konzepten der "Normalisierung" und des "Normalismus". Laut Link (ebd.: 281) versteht man darunter, dass Subjekte durch bestimmte Formen der Macht, v. a. der sogenannten "Disziplinarmacht", geformt, korrigiert, und reguliert werden; dadurch werden Normalitäten in komplexen und verdateten Gesellschaften hergestellt. Solche Normalitäten können sich nun verändern; beispielsweise können sich die Konnotationen von Begriffen verändern, von eher positiven zu eher negativen Mitbedeutungen, und umgekehrt; Begriffe werden also rekontextualisiert.

Es können auch Forderungen, die zunächst von marginalisierten Gruppierungen vorgebracht werden, von Mainstream-Parteien im Laufe gesellschaftlicher Veränderungen aufgegriffen und umgesetzt werden, derart zur Normalität mutieren. Dies ist ein durchaus gängiger Prozess, der häufig in den oben erwähnten Kämpfen um hegemoniale Bedeutungsmacht auftritt. Die Grenzen sind nirgends ein für alle Mal festgelegt, wie sich an der zurzeit vor allem in der westlichen Welt erfolgten Liberalisierung gleichgeschlechtlicher Partnerschaften und Ehen leicht nachvollziehen lässt. Ebenso veränderten sich im Laufe eines Prozesses der Normalisierung Migrationsbestimmungen: Waren die Grenzen mancher europäischer Nationalstaaten vor dem Fall des Eisernen Vorhangs 1989 noch recht durchlässig, wenn es um die Aufnahme von Flüchtlingen ging, so wurden diese nach 1989 recht dicht verschlossen, sowohl Flüchtlingen wie MigrantInnen gegenüber (Matouschek et al. 1995; Reisigl/Wodak 2000, 2001; Wengeler 2003a,b; Wodak/Boukala 2014, 2015; Wodak 2015). Normalitäten ändern sich also kontinuierlich und werden dann auch akzeptiert.

\section{Neoliberalismus, Populismus und kritische Öffentlichkeit}

Nach diesen sehr gerafften Hinweisen zu einigen Funktionen und Wirkungen des politischen Diskurses will ich mich drei zentralen politischen Faktoren zuwenden, die - wie ich meine heutzutage unser Denken vielfach bestimmen und in einem Spannungsfeld zueinander stehen, nämlich Neoliberalismus, Populismus und kritische Öffentlichkeit. Gerade diese spielen eine wichtige Rolle, wenn man Prozesse der Normalisierung in unseren heutigen Gesellschaften genauer verstehen und erklären will. Die Rekontextualisierung von Begriffen, Argumenten und Ideologemen erfolgt, so meine ich, von einer Öffentlichkeit in die andere, also von der Politik über die Medien in unseren Alltag; eine zunächst ökonomische Theorie, wie der Neoliberalismus, verliert ihre enge Fach- und disziplinäre Bedeutung und wird als Schlagwort und Slogan vielfach umbedeutet (vgl. Wodak/Fairclough 2010). Auch der Begriff "Populismus" dient häufig einer generellen oberflächlichen Charakterisierung politischer Programme und Parteien, obwohl, wie weiter unten ausgeführt, sicherlich jede/r PolitikerIn populistische Elemente in ihre oder seine Rhetorik notwendigerweise miteinbaut.

Will man also die Karriere eines Konzepts, wie Integrationsunwilligkeit dekonstruieren, so werden alle diese Faktoren in jeweils kontextabhängiger Form relevant: welche Parteien bemächtigen sich dieses Begriffs, wann und warum? In welcher Weise beeinflussen auch neoliberale Elemente die Debatten zur Einwanderungspolitik; haben sich die Debatten in den letzten Jahrzehnten, seit 1989, verändert? Und schließlich, welche Öffentlichkeiten bemächtigen sich in welcher Weise dieses Begriffs, in welcher Absicht, und mit welchem Effekt?

Anhand der quantitativen und qualitativen Analyse des Begriffs "Integrationsunwilligkeit" will ich im Weiteren empirisch aufzeigen, wie dieses undefinierte vage Konzept sowohl neo- 
liberal wie populistisch vielfach in den Medien und in der Politik affektiv aufgeladen wird und wie man es im Sinne einer kritischen Öffentlichkeit hinterfragen muss. Der Begriff, der in Österreich zunächst seit den 1990erjahren als Indikator für rechtspopulistische Einstellungen diente, wandert nun, so kann man allgemein und auch statistisch fundiert beobachten, seit einigen Wochen und Monaten in die Mitte des politischen Spektrums und wird zum metonymischen Symbol einer veränderten, restriktiven Migrationspolitik.

\subsection{Neoliberalismus}

Wenn man an Neoliberalismus denkt, so fällt vielen sicherlich sofort der berühmte Satz der ehemaligen britischen Politikerin Margaret Thatcher ein, nämlich:

What is wrong with the deterioration? I think we have gone through a period when too many children and people have been given to understand "I have a problem, it is the Government's job to cope with it!" or "I have a problem, I will go and get a grant to cope with it!" "I am homeless, the Government must house me!" and so they are casting their problems on society and who is society? There is no such thing [as society]! There are individual men and women and there are families and no government can do anything except through people and people look to themselves first.

(www.margaretthatcher.org/document/106689 [05.11.2015])

Jeder ist also für sich selbst verantwortlich; und was man nicht schafft, liegt nicht an irgendwelchen Strukturen, sondern man muss es sich selbst zuschreiben. Diese Grundhaltung stammt natürlich aus der neoliberalen Ökonomie und wurde auf die gesamte Gesellschaft und alle ihre Bereiche übertragen: die Idee eines selbst regulierenden Marktes, der nicht mehr in umfassende Sozialbeziehungen eingebettet ist (wie dies Karl Polanyi treffend beschrieb); die Idee eines selbst regulierenden Marktes ohne Intervention und Kontrolle staatlicher Institutionen. Wettbewerb und Leistung, ranking und benchmarks charakterisieren unseren Alltag in der sogenannten Wissensgesellschaft und knowledge-based economy (übrigens natürlich auch an den Universitäten). Der britische Soziologe Bob Jessop (2008) trifft mit der folgenden Definition "ins Schwarze":

Every economy is a knowledge economy but not every economy has been called a knowledge economy, let alone finds itself so labelled by its most prominent spokespersons as one of its most significant contemporary self-descriptions [....] Related theoretical paradigms seek to establish the novelty of the KBE by identifying its distinguishing features in terms of some combination of the reflexive application of knowledge to the production of knowledge, the key role of innovation, learning, and knowledge transfer in economic performance, and the increasing importance of the intellectual commons and/or intellectual property rights in contemporary competition. In turn the hegemonic policy paradigm is especially concerned to establish the reality of the KBE through the compilation and repetition of statistical indicators, through the development of benchmarks and league tables, and through the elaboration of an interwoven set of useful concepts, slogans, and buzzwords. These can then be applied to generate a relatively simple set of policy prescriptions and legitimations to be applied to many sectors, many scales, and many countries.

(Jessop 2008: 13)

Wesentliche gesellschaftliche Bereiche und entsprechende Maßnahmen haben - Jessop zufolge - seit den 1990erjahren innerhalb der Europäischen Union beispielsweise die Werte, Wertungen, und damit auch den Wortschatz der KBE übernommen (vgl. Muntigl et al. 2000). Das wichtigste Dokument wurde 1996 von der OECD publiziert, mit dem Titel "The Knowledge- 
Based Economy". 1997 folgten die Richtlinien für den Wettbewerb ("competitiveness") in der Form von "National Innovation Systems". Auch innerhalb der Europäischen Union führte dies zur Einrichtung der "Competitiveness Advisory Group" (CAG), die Richtlinien und Policies gegen Arbeitslosigkeit auf der Grundlage der KBE ausarbeiten sollte (vgl. Wodak 2000). "Knowledge Management" wurde zur Schlüsseldisziplin, entsprechende Ministerien wurden errichtet, und Nationalstaaten begannen, ihre nationalen Innovationssysteme (NIS) zu entwerfen. Das Lexikon wurde alsbald entsprechend standardisiert (vgl. American National Standards Institute und Global Knowledge Economics Council 2001). "Competitiveness indexes" wurden berechnet und ausgegeben, z. B. der "Global Competitiveness Report" (World Economic Forum) seit 1979 und das "World Competitiveness Yearbook" (herausgegeben von dem Institute for Management Development in Genf) seit 1989.

Heutzutage begegnen wir daher einer globalen Industrie, die Wettbewerbskriterien vorgibt, Rankings berechnet und für die global players entscheidende Richtwerte produziert (vgl. Jessop/Fairclough/Wodak 2008). Die OECD publiziert jährliche Berichte; neue Konzepte und Begriffe wurden dafür entworfen (vgl. Godin 2006: 19, 24). Godin (2004: 684) weist v. a. auf die vielen Graphiken und Statistiken hin, die ein Eigenleben gewonnen haben und gewinnen, obwohl natürlich solche Indikatoren der Qualität und Dynamik von Wissen und Innovation niemals gerecht werden können. Sehr überzeugend weist Jessop (2008) daraufhin, dass die KBE als politische und ökonomische Strategie gerade auch durch den von ihr produzierten Diskurs sich ständig neu herstellt und damit zur überragenden ökonomischen und gesellschaftlichen Ideologie geworden ist. Dieser neue Diskurs drang und dringt entsprechend auch in die Bildungsinstitutionen ein; neue Konzepte wie "lebenslanges Lernen", "Flexibilisierung", "benchmarks", "human resources" usw. haben sich durchgesetzt.

Ebenso ist der Diskurs über Integration und Migration von solchen neoliberalen Versatzstücken charakterisiert und bewegt sich zwischen zwei Extremen: Migration soll entweder gestoppt werden, da MigrantInnen - so wird behauptet - unsere Gesellschaft und sozialen Einrichtungen ausnützen und unsere Arbeitsplätze wegnehmen, ohne etwas beizutragen; oder: Migration ist willkommen, wenn es nachweisbare Leistungen gibt - also, wie der aktuelle hegemoniale politische Slogan lautet: "Integration durch Leistung"! Es bleibt allerdings unklar, wieviel und was genau geleistet werden muss.

Der Diskurs über Migration und MigrantInnen ist ökonomisiert und wird unkritisch von vielen akzeptiert; es geht nicht um Menschen, sondern um finanzielle Vor- oder Nachteile für "unsere Gesellschaft". Das Nutzensargument (oder -Topos; siehe unten) lässt sich vor allem seit den 1990erjahren feststellen; früher, als sich die Debatte um die sogenannten Gastarbeiter drehte, wurden vor allem kulturelle Argumente ins Treffen geführt, wie beispielsweise, dass diese nicht "zu uns" passten; man hatte ja die Gastarbeiter quasi eingeladen, sie also geholt, weil sie bereit waren, niedrig bezahlte Arbeiten, die "wir" nicht machen wollten, zu übernehmen; und dann war man überrascht, als sie nicht einfach wieder verschwanden, sondern sich "bei uns" wohlfühlten und niederließen (Wodak/Matouschek 1993; Wengeler 2003a, b).

Heutzutage dreht es sich aber nicht um gewollte und eingeladene Fremde, sondern um andere, die 'anders ausschauen' und die gekommen sind, ohne dass man sie holte. In diesem Fall sind, wie der britisch-polnische Soziologe Zygmunt Bauman (1995) pointiert behauptet, zwei Re- 
aktionen zu beobachten: entweder die Aufnahmegesellschaft schluckt die Fremden (sie assimilieren sich); oder sie spuckt sie aus (sie assimilieren sich nicht).

\subsection{Populismus}

Der ökonomistische Diskurs der Politik wird affektiv aufgeladen und in populistischer Weise von unterschiedlichen Parteien und Positionen benutzt. Dazu muss ich nochmals betonen: Jeder und jede PolitikerIn ist notwendigerweise populistisch, denn jede und jeder will ja viele ansprechen und für viele sprechen. Populismus als solcher ist ein rhetorischer Stil, der den "kleinen Mann und die kleine Frau", das Volk, gegen die "da oben" stellt; eine Rhetorik, die sich an das Volk wendet und vorgibt, für das Volk zu sprechen.

Rechts- oder links-populistisch wird eine solche Rhetorik nur dann, wenn sie mit ganz bestimmten Inhalten aufgeladen wird - etwa mit genauen Definitionen, wer nun das "echte Volk'" sein soll, für das eingetreten wird; welche die genauen Ziele sein sollen, die anscheinend dem Volk am Herzen liegen, usw. (vgl. Wodak 2013a, b, 2014b, 2015; Wodak/KhosraviNik/Mral 2013). Der österreichische Politikwissenschaftler Anton Pelinka (2013: 9) bietet eine umfassende Definition des heutigen Rechtspopulismus aus sozialwissenschaftlicher Sicht an:

[...] contemporary populism does not so much mobilize against the (perceived) enemy above but more against the (perceived) enemy from abroad. Populism has become more and more ethnonationalistic. Populist anti-élitism today is directed against those who seem to be responsible for Europeanization and globalization, and especially for mass migration, against élites who have opened the doors to foreign influence and to foreigners. [...] And, of course, the tendency to see individuals (politicians - the "classe politica", or intellectuals - "the chattering classes") as responsible for modernizing trends is beyond any realistic and empirically sound analysis of the trend which tends to put an end to the nation state.

(Pelinka 2013: 9)

$\mathrm{Zu}$ dieser inhaltlichen Beschreibung kommen nun die pragmatischen, argumentativen und rhetorischen Elemente hinzu, die erst "in der Integration von Form und Inhalt" den persuasiven Appell an das so konstruierte "Volk" erst entstehen lassen (siehe weiter unten). Im Falle der Integration/Migration bedeutet dies, dass genau definiert wird, wer zu "uns" darf, und wer nicht; was "uns" nützt und was nicht; wovor man Angst haben muss, und wovor nicht; wer an was schuld ist; usw. Insofern können wir sowohl die ideologischen Versatzstücke des Rechtswie Linkspopulismus relativ genau anhand einer linguistischen Analyse erkennen und analysieren. ${ }^{1}$

1 Die Differenzierung zwischen Rechts- und Linkspopulismus wird häufig durch den überschäumenden und häufig rassistischen Nationalismus des Rechtspopulismus und die internationale Orientierung des LinksPopulismus charakterisiert. Allerdings, so meint die Politikwissenschaftlerin Chantal Mouffe (2015: 8), müsse man zwischen Bürgerbewegungen ohne Infrastruktur und historischer Tradition (beispielsweise den sogenannten spanischen "Indignados"), und links-populistischen Bewegungen, wie etwa der griechischen Syriza, die auf der Jahrzehnte-alten griechischen Linke basiert, unterscheiden. So bestehe bei der Front National in Frankreich "das Volk" aus den "guten, eingeborenen, biologischen Franzosen", während bei den Linken "die Migranten Teil der Arbeiterklasse, des ,Wir"' seien (ebd.). 


\subsection{Kritische Öffentlichkeit}

Und schließlich muss noch der Begriff der "kritischen Öffentlichkeit" kurz diskutiert werden: Ist die neue deutsche (und österreichische) Bewegung "Pegida" etwa eine kritische Öffentlichkeit? Die spanischen Indignados? Die Ägypter am Tahrir-Platz? Ein Internetforum? Vom Forum Romanum also zum Internetforum? Wir sind ja mit vielen Öffentlichkeiten konfrontiert, auf der Straße, im Internet, im Web 2.0 und in den Medien (Angouri/Wodak 2014; Koller/Wodak 2008; Triandafyllidou/Wodak/Krzyżanowski 2009). Zu dieser signifikanten Veränderung unserer Wahrnehmung von Öffentlichkeit meint Habermas (1998: 66-67) pointiert:

Erst zu Beginn des 20. Jahrhunderts verdichten sich Massenströme, Massenorganisationen und Massenaktionen zu aufdringlichen Erscheinungen, die die Vision vom Aufstand der Massen (Ortega y Gasset) auslösen. In der Massenmobilisierung des Zweiten Weltkriegs und im Massenelend der Konzentrationslager entfaltet sich ebenso wie nach 1945 im Massentreck der Flüchtlinge und im Massenchaos der displaced persons ein Kollektivismus, der sich auf dem Titelbild von Hobbes Leviathan angekündigt hatte: schon dort sind die zahllosen Einzelnen anonym zur übermächtigen Gestalt eines kollektiv handelnden Makrosubjekts verschmolzen. Aber seit der Mitte des Jahrhunderts verändert sich die Physiognomie der großen Zahlen. Die Präsenz versammelter, in Marsch gesetzter oder zusammengepferchter Körper wird abgelöst von der symbolischen Inklusion des Bewusstseins der Vielen in immer weiter ausgreifende Kommunikationsnetze: die konzentrierte Masse verwandelt sich ins zerstreute Publikum der Massenmedien. Die physischen Verkehrsströme und Verkehrsstaus schwellen weiter an, während die elektronische Vernetzung der individuellen Anschlüsse die auf Straßen und Plätzen zusammengeballten Massen zum Anachronismus macht.

(Habermas 1998: 66-67)

Das Auftreten kritischer Öffentlichkeiten der europäischen Neuzeit können wir zunächst auf die Aufklärung zurückführen, auf die sogenannte "Republik der Briefe", im 17. und 18. Jahrhundert, wo Philosophen einander Briefe schrieben und in Dialog traten. Habermas lenkt unseren Blick auf das Erwachen aufmüpfiger bürgerlicher Öffentlichkeiten in den Kaffeehäusern, Salons und Geheimgesellschaften Europas im 18. und 19. Jahrhundert. Es dauerte lange, bis sich diese intellektuellen, geschlossenen Zirkel öffneten, beispielsweise Frauen gegenüber, und allen interessierten BürgerInnen, und nicht nur auf einige wenige Eliten beschränkt blieben.

Eine kritische Öffentlichkeit ist nicht jede Öffentlichkeit, sondern - ganz im Sinne der angesprochenen Tradition - eine, die einen offenen Austausch zulässt; die sich also nicht dogmatisch verschließt und jene, die anderer Meinung sind, vornweg diffamiert; damit ist eine normative Ebene notwendigerweise integriert. Daher ist also meiner Meinung nach eine Öffentlichkeit wie Pegida, die die Medien in generalisierter Form als "Lügenpresse" bezeichnet und Menschen mit anderen Meinungen insgesamt als "Volksverräter" tituliert, keine kritische Öffentlichkeit. Ihr fehlt unter anderem die Fähigkeit zum offenen und respektvollen Austausch nicht zuletzt auch über die eigenen Ansichten. Sind wir hier etwa mit "Newspeak" oder sogar "Oldspeak" konfrontiert? 


\subsection{Der diskurshistorische Ansatz}

Zum Abschluss meiner Überlegungen zum gegenwärtigen politischen Diskurs dient eine Diskursanalyse des Begriffs Integrationsunwilligkeit als Fallbeispiel: Integrationsunwilligkeit beherrscht momentan die österreichischen Medien und die Politik, ein Begriff, der über viele Jahre ausschließlich von der Freiheitlichen Partei Österreichs (FPÖ) (gemeinsam mit dem Begriff Integrationsverweigerung) gebraucht wurde und nun in die politische Mitte gelangt ist, also "normalisiert" wurde, vom Mainstream angenommen wurde.

In der Diskursforschung gehen wir, ganz im Sinne von Orwell und Laclau, davon aus, dass politisches Handeln vor allem kommunikativ ist und sich nicht nur in Reden von PolitikerInnen erschöpft. Aufgrund unterschiedlicher institutioneller Bedingungen und einer Bandbreite von RezipientInnen werden je spezifische "Genres" zur Umsetzung bestimmter politischer Interessen verwendet. Politischer Diskurs im weitesten Sinn besitzt persuasiven Charakter, der mehr oder weniger explizit oder implizit manifest wird; deshalb greifen auch heutzutage viele Genres des politischen Alltags auf die Figuren und Stilmittel der klassischen Rhetorik zurück. Das Genre (die Textsorte), also schriftlicher oder mündlicher Text, Rede oder Bescheid, Bild oder SMS, impliziert notwendigerweise jeweils bestimmte argumentative wie auch sprachliche Verfahren.

Theoretisch und methodisch liegt der vorliegenden Arbeit der Diskurshistorische Ansatz (DHA) der Kritischen Diskursanalyse/Critical Discourse Analysis (CDA) zugrunde (Reisigl 2014; Wodak/Meyer 2009). Die CDA ist - allgemein gesehen - ein problemorientiertes, trans- und interdisziplinäres Forschungsprogramm. Für die Analyse von öffentlichen, insbesondere politischen Diskursen ist dabei die Einbeziehung der historischen Dimension besonders wichtig, die eine umfassende, multi-perspektivische Interpretation von Texten erst ermöglicht, indem der konkrete Kontext der Textproduktion mit berücksichtigt und so intertextuelle, interdiskursive, diachrone wie synchrone Querverbindungen ermöglicht werden. Operationalisiert wird ein solches kontext-sensitives Vorgehen durch die Konzepte der Intertextualität, Rekontextualisierung und Interdiskursivität. Diese begriffliche Trias definiere ich kurz zusammengefasst wie folgt (vgl. Reisigl/Wodak 2009; Wodak 2011, 2015):

Texte stehen immer in größeren Zeit- und Raumzusammenhängen, synchron und diachron, sind daher niemals als isolierte Informationsäußerungen $\mathrm{zu}$ verstehen; dies wird mit Intertextualität erfasst. Diskurse überlappen einander, Texte und Genres sind daher oft hybrid. So beinhalten Diskurse über Beschäftigungspolitik beispielsweise oft Argumente und Topoi aus Diskursen über Soziales oder über Migration. Diese Phänomene werden als Interdiskursivität bezeichnet. Rekontextualisierung beschreibt, wie sich Topoi und Argumente im Transfer von Genre zu Genre und Öffentlichkeit zu Öffentlichkeit verändern, wie sie damit neue Bedeutungen gewinnen - d. h. welches Eigenleben sie dabei entwickeln.

Über eine detaillierte, qualitative und quantitative Analyse des Diskurses und der Texte erfahren wir Informationen über die immanenten Ideologien und Einstellungen. Diskurse sind sowohl thematisch (durch Bezug auf ein Makrothema), multiperspektivisch (d. h. dialogischargumentativ) wie auch von der Textsorte, dem Genre, stark bestimmt und werden in einzel- 
nen Texten realisiert. Jeder Text bezieht sich immer auf andere Texte, diachron und synchron gesehen (siehe oben). Analytisch wird in der diskurshistorischen Methode zwischen drei ineinander verwobenen Analysedimensionen unterschieden, nämlich zwischen

1. Inhalten;

2. Argumentationsstrategien und Topoi; und

3. sprachlichen Realisierungsmitteln beziehungsweise Realisierungsformen.

An dieser Stelle würde es natürlich zu weit führen, alle linguistischen Kategorien im Einzelnen anzuführen bzw. auch die Grammatiktheorien, die solchen Kategorien zugrunde liegen, zu präsentieren. Auch hier muss ich auf Reisigl/Wodak (2001, 2009) verweisen. In der folgenden Analyse des Begriffs "Integrationsunwilligkeit" beziehe ich mich vor allem auf Elemente der klassischen Rhetorik und Argumentationstheorie wie von Kienpointner (1992) und Wengeler (2003a, b) dargestellt (siehe Reisigl 2014). Die korpuslinguistische Analyse beruht auf Baker et al. (2008). Um Einstellungen und Ideologien, Identität und Fragmentierung, Brüche und Widersprüche zu untersuchen, beziehe ich mich im vorliegenden Aufsatz u. a. auf folgende Fragestellungen:

- Wie werden Sachverhalte und soziale Akteure benannt bzw. wie wird auf Sachverhalte und soziale Akteure Bezug genommen?

- Welche Eigenschaften und Charakteristika werden den betreffenden Sachverhalten und Akteuren zugeschrieben?

- Welche Argumente bzw. Argumentationsschemata werden herangezogen, um die Charakterisierung von spezifischen Personen oder Gruppen von Personen bzw. bestimmte Handlungen jener Personen zu rechtfertigen und zu legitimieren? Welche Präsuppositionen liegen diesen Annahmen zugrunde, welche Implikaturen werden sichtbar?

Diesen Fragestellungen entsprechen drei Gruppen von diskursiven Strategien:

- Referentielle Strategien

- Prädikative Strategien

- Argumentative Strategien

Referentielle Strategien stellen Strategien zur linguistischen Konstruktion, Identifikation und Repräsentation von Sachverhalten und sozialen Akteuren dar, letztere beispielsweise in Form von Wir-Gruppen und Sie-Gruppen. Diese Strategien werden in erster Linie über Nominalisierungen linguistisch realisiert, allerdings sind gerade im Deutschen auch andere Realisierungsformen (Adjektive, Präpositionalphrasen, usw.) von Bedeutung.

Strategien der Prädikation sind Strategien, mittels derer den zuvor identifizierten Sachverhalten oder sozialen Akteuren - seien es Individuen oder Gruppen von Personen - positive oder negative Eigenschaften in Form von impliziten oder expliziten und mehr oder weniger wertenden Prädikaten zugewiesen werden. Prädikative Strategien können in verschiedener Form linguistisch realisiert werden. Dazu zählen u. a. spezifische Formen der Referenz und der damit einhergehenden Konnotation bzw. Denotation; Attribute, d. h. Adjektive, Appositionen, 
Präpositionalphrasen, Relativsätze u. a.; Prädikate; explizite Vergleiche und Gleichsetzungen; Metaphern; rhetorische Figuren wie Metonymien, Euphemismen, usw.; Präsuppositionen.

Argumentative Strategien/Topoi sind Strategien, die u. a. dazu dienen, die jeweilige Charakterisierung von spezifischen Personen bzw. Gruppen von Personen zu rechtfertigen und zu legitimieren. Als Topoi bezeichnen wir inhaltsbezogene Schlussfolgerungsregeln, die ein oder mehrere Argumente mit der Schlussfolgerung verknüpfen (obligatorische explizite oder erschließbare Prämissen im Rahmen der Argumentation) (vgl. Reisigl 2014; Wodak 2014a). Bei der Analyse von "Integrationsunwiligkeit" in den österreichischen Medien kommen vor allem folgende Topoi zur Geltung (Tabelle 1):

\begin{tabular}{|c|c|}
\hline Topos & Beschreibung \\
\hline Topos des Volkes & $\begin{array}{l}\text { Wenn das Volk eine Handlung will/nicht will, dann soll diese } \\
\text { Handlung erfolgen/nicht erfolgen. }\end{array}$ \\
\hline $\begin{array}{l}\text { Topos von Recht und } \\
\text { Ordnung }\end{array}$ & $\begin{array}{l}\text { Wenn ich/wir die Macht haben, dann garantiere/n ich/wir für } \\
\text { Recht und Ordnung }\end{array}$ \\
\hline Kulturtopos & $\begin{array}{l}\text { Weil die Kultur einer bestimmten Gruppe von Leuten ist, wie sie } \\
\text { ist, entstehen spezifische Probleme in spezifischen Situationen. }\end{array}$ \\
\hline Belastungstopos & $\begin{array}{l}\text { Wenn eine Person, eine Institution oder ein "Land" durch spezi- } \\
\text { fische Probleme belastet wird, dann sollten Maßnahmen ergrif- } \\
\text { fen werden, um diese Belastung zu verringern. }\end{array}$ \\
\hline $\begin{array}{l}\text { Topos der demokrati- } \\
\text { schen Mitbestimmung }\end{array}$ & $\begin{array}{l}\text { Wenn ich/wir die Macht haben, dann wird das Volk demokra- } \\
\text { tisch mitbestimmen. }\end{array}$ \\
\hline Nutzen-Topos & $\begin{array}{l}\text { Wenn eine bestimmte Handlung aus einer spezifischen Sicht als } \\
\text { nützlich erscheint, dann sollte sie durchgeführt werden. }\end{array}$ \\
\hline
\end{tabular}

Tabelle 1: Relevante Topoi in Debatten zu "Integrationsunwilligkeit"

In rechts- wie linkspopulistischer Manier (siehe oben) haben sich diverse PolitikerInnen des Begriffs bemächtigt, ohne ihn je zu definieren; er ist affektiv aufgeladen, denn jede und jeder verbindet damit notwendigerweise etwas Böses und assoziiert je nach Bedarf unterschiedliche, abzulehnende Verhaltensweisen von Fremden - ein geradezu ideales Beispiel für einen floating signifier und ein vages Konzept, das durchaus im Sinne von Orwells Doublethink ganz unterschiedlich definiert werden kann.

\subsection{Quantitative und qualitative Analyse des Begriffs "Integrationsunwilligkeit"}

"Integrationsunwilligkeit" bietet sich für alle möglichen Bedeutungen an. Wir haben im Zuge des FWF Projekts zur "Diskursiven Konstruktion der Österreichischen Identität 2015" 
(http://pf.fwf.ac.at/de/wissenschaft-konkret/project-finder/33284) ein umfassendes Korpus von 72 Artikeln aus den überregionalen österreichischen Tageszeitungen im Zeitraum von 20.01.2015 bis 03.02.2015 zusammengestellt (gefiltert nach der Häufigkeit der Verwendung des Begriffs "Integrationsunwilligkeit" als Makrothema) und sowohl quantitativ als auch qualitativ nach Frequenz und Kollokationen untersucht. Eine Kollokationsanalyse meint die genaue Analyse der jeweiligen Kontexte, in denen der Begriff auftaucht (vier Worte vor und vier Worte nach dem untersuchen Begriff).

Dabei haben wir alle in diesem Zeitraum erschienenen Zeitungsartikel nach dem Begriff "Integrationsunwilligkeit" durchsucht und anhand von "Google-Analytics" mit dem Gesamtkorpus von Medienberichten seit 1990 verglichen. Dadurch konnten wir einerseits die Frequenz des Begriffs erfassen wie auch andrerseits den diachronen Verlauf nachzeichnen. Durch ein solches Verfahren war es möglich, in statistisch signifikanter Weise die wesentlich größere Häufigkeit der Begriffsverwendung in dem Beobachtungszeitraum festzustellen; gleichzeitig konnten wir die jeweiligen referentiellen und prädikativen Strategien analysieren, d. h. ausloten, wer diesen Begriff in welchem Zusammenhang zu welchem Zeitpunkt und Anlass benutzt hatte. ${ }^{2}$

Die 72 Texte erweisen die - im oben beschriebenen Vergleich - plötzliche Popularität der bisher selten gebrauchten Begriffe Integrationsunwillige (64) und Integrationsunwilligkeit (60), gegenüber den schon länger im Umlauf befindlichen Begriffen Integrationsverweigerer (15) und Integrationsverweigerung (21), sowie den besonders eigenwilligen Desintegrationstendenzen (1), Ausländerintegration (1), Nichtintegration (1) und sogar Integrationsmuffel (2). Der Fokus hat sich also von einer "Verweigerung", die zumindest dem Konzept nach noch etwas tatsächlich Getanes oder nicht Getanes meint, hin zu einem "Unwillen", auf den bestenfalls rückgeschlossen werden kann, verlagert.

Ordnet man die Verwendung der Begriffe konkreten SprecherInnen innerhalb der Domänen Politik und Medien zu (schließt also anonyme Postings in Tageszeitungen etwa aus), so zeigt sich, dass diese bis zur aktuellen Debatte entweder von VertreterInnen bzw. Mitgliedern der FPÖ verwendet und zitiert werden (bis 2010 ausschließlich), danach zunehmend im Boulevard, und ab Mitte 2014 immer mehr im gesamten politischen Spektrum. Die Zuordnung des Begriffs zu SPÖ und ÖVP, bzw. konkreten Sprechern wie Bundesminister Sebastian Kurz, Landeshauptmann Hans Niessl und Landeshauptmann Franz Voves, lässt sich mit der Analyse der Kollokate des Begriffes "integrationsunwillig" verdeutlichen (Tabelle 2):

\footnotetext{
2 Vgl. www.google.com/intl/de_ALL/analytics/features/index.html [05.11.2015] zu den Funktionen von Google Analytics. Es wurde darauf verzichtet (außer für eine Pilotstudie), ein Vergleichskorpus zu erstellen, da unser Forschungsinteresse auf einen einzigen Begriff fokussiert war, dessen statistisch signifikantere Verwendung durch eine Pilotanalyse im Vergleich zur Verwendung im gleichen Zeitraum 2014 eindeutig erwiesen war. Es geht ja bei dieser Untersuchung nicht um eine korpuslinguistische Studie, sondern um eine linguistisch fundierte Begriffsgeschichte.
} 


\begin{tabular}{|l|l|}
\hline Kollokat & Frequenz \\
\hline Strafen (einschl. Verwaltungsstrafen) & 46 \\
\hline Schüler & 25 \\
\hline SPÖ & 20 \\
\hline ÖVP & 17 \\
\hline Sozialdienst für & 14 \\
\hline Vorgehen gegen & 12 \\
\hline Strafrechtlich & 10 \\
\hline Ahnden & 10 \\
\hline Sanktionen & 9 \\
\hline Eltern & 6 \\
\hline Mangel (einschl. mangelnd) & 5 \\
\hline Forderung & 5 \\
\hline Tatbestände & 4 \\
\hline
\end{tabular}

Tabelle 2: Häufigste Kollokate

Dabei zeigt sich, dass es in den Texten vorwiegend um Geldstrafen bzw. das Bestrafen von Schülern und Eltern geht, um ein Vorgehen gegen etwas, das Ahnden von Mängeln, Sanktionen gegen jemanden bis hin zur Abschiebung, um Forderungen nach gesetzlichen Bestimmungen und dergleichen mehr. Um Integration im positiven Sinne geht es in der aktuellen Debatte mit Sicherheit nicht. Definiert wird der Begriff nirgends und von niemandem. Die Word Cloud verdeutlicht aufgrund der quantitativen Auswertung die Dominanz der negativen Sanktionen, die mit "Integrationsunwilligkeit" verbunden werden (siehe Word $\mathrm{Cloud}^{3}$ ).

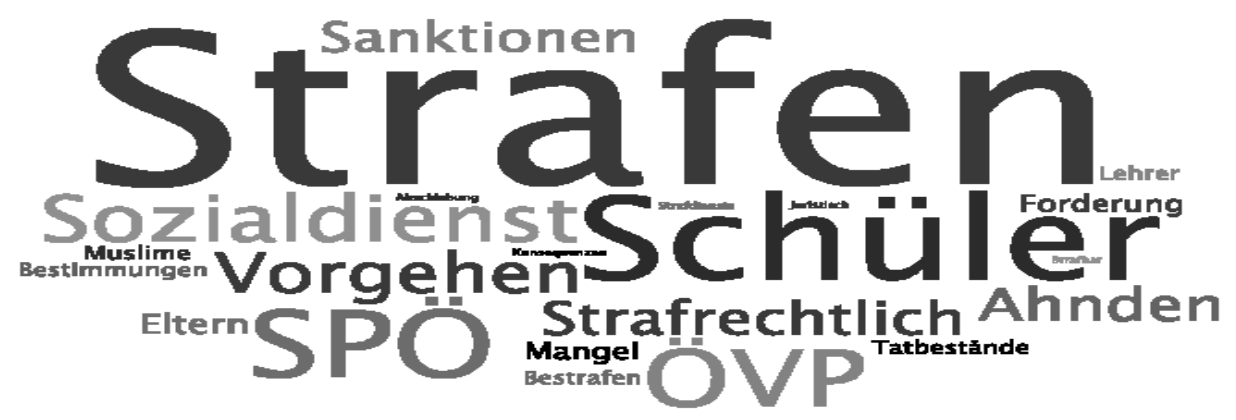

Abbildung 1: Quelle: www.jasondavies.com/wordcloud/\#\%2F\%2Fwww.jasondavies.com\% 2Fwordcloud\%2Fabout\%2F [05.11.2015]

3 Word Clouds erlauben in korpuslinguistischen Analysen eine anschauliche, graphische Umsetzung von Begriffsfrequenzen, vgl. www.jasondavies.com/wordcloud/ $\# 2 \%$ F $\% 2 \mathrm{Fwww}$.jasondavies.com\%2Fwordcloud $\% 2$ Fabout $\% 2 F)$ [05.11.2015]. 
Beispiele aus dem Korpus gibt es viele. Im Folgenden seien drei typische Textstellen wiedergegeben, die die oben angeführten Topoi illustrieren mögen:

Text 1: Kulturtopos, Topos von Recht und Ordnung

[...] Es sei nicht akzeptabel, dass sich ein Bursche von einem weiblichen Pädagogen nichts sagen lasse, sich ein Vater weigere, bei einem Sprechtag einer Lehrerin die Hand zu geben oder die Tochter nicht in den Schwimmunterricht schicke. "Das ist ein so massiver Bruch mit und ein Angriff auf unsere Grundwerte, dass wir nicht wegschauen dürfen. Lehrern muss möglich sein, pädagogische Maßnahmen zu verordnen." Welche? "Ein verpflichtendes Gespräch mit einem Schulpsychologen und am Schulstandort Dienst an der Allgemeinheit." Was wäre das? "Eine schulgemeinschaftliche Arbeit während der Freizeit." Das wäre besser als das, was derzeit möglich sei: "Dass Schüler, mit denen es Probleme gibt, von der Schule geschmissen und als 'unbeschulbar' abgestempelt werden. Mehr Durchgriffsrechte für Lehrer sind nötig." Zielt das, angesichts der von ihm genannten Beispiele, nur auf muslimische Schüler ab? "Das gilt für alle, die Werte verletzen." [...]

(Kurier 2015; Hervorhebungen RW) ${ }^{4}$

Die Topoi lassen sich wie folgt zusammenfassen:

Wenn Burschen sich bestimmten Konventionen nicht fügen und Mädchen nicht in den Schwimmunterricht gehen, dann passen sich diese Jugendlichen "unserer Kultur" nicht an.

Brüche mit/Angriffe auf "unsere Grundwerte" implizieren, dass man nicht wegschauen dürfe.

Wenn "unsere Werte" verletzt werden, dann muss stärker durchgegriffen werden.

Text 2: Kulturtopos, Topos von Recht und Ordnung

ÖVP-Klubobmann Reinhold Lopatka hat sich Dienstagabend in Graz bei einer Diskussion über den Islam "für Verwaltungsstrafen" bei Integrationsunwilligen in Österreich ausgesprochen, wenn Freiwilligkeit nicht ausreiche: "Das spürt man dann auch."

(Der Standard 2015; Hervorhebungen RW)

Text 3: Topos des Nutzens, Topos von Recht und Ordnung

Unmittelbar nach den Anschlägen von Paris eröffnete der im Wahlkampf stehende steirische Landeshauptmann Franz Voves (SPÖ) die Diskussion, in dem er auch strafrechtliche Konsequenzen für "Integrationsunwillige" einforderte. Unterstützung signalisierte Burgenlands Landeshauptmann Hans Niessl (SPÖ), der zuerst im KURIER höhere Verwaltungsstrafen forderte, wenn Eltern beispielsweise nicht zu Sprechtagen kommen und Vorladungen ignorieren.

(Kurier 2015; Hervorhebungen RW)

Gerade in der emotionalen Vagheit wird der Begriff gefährlich: denn er dient nun dazu, manche ein- und andere auszuschließen, ohne dass klare und distinktive Kriterien angegeben werden können. Verfolgt man die rezente Debatte, so erweist sich, dass der Begriff unmittelbar nach den schrecklichen Anschlägen in Paris von 07. und 09. Jänner 2015 seinen Höhenflug in der österreichischen Politik beginnt und sich vor allem auf das Feld der Schule bezieht. Auch zeigt sich, dass der Begriff erst dann, als er in der "Mitte" angekommen ist, also "normali-

4 "Bildung gegen Extremismus" - auf das setzt SPÖ-Unterrichtsministerin Gabriele Heinisch-Hosek. Auch an den Pädagogischen Hochschulen soll Prävention gelehrt werden". 
siert" wurde, indem er von SPÖ und ÖVP-Politikern aufgegriffen wurde, im Diskurs eine nennenswerte Verbreitung finden konnte (Abbildung 2; erstellt aufgrund von GoogleAnalytics der gesamten österreichischen Presseberichterstattung).



Abbildung 2: Verbreitung 2005-2015

\subsection{Die Vermengung zweier Diskursstränge}

Mehrere Lesarten und Erklärungen bieten sich an: Der bevorstehende Wahlkampf in den Bundesländern Steiermark und Burgenland, im Frühjahr 2015. Der Wahlkampf führt dazu, dass sozialdemokratische Landeshauptmänner zunehmend einen rechtspopulistischen Diskurs zur Migration einnehmen. Und gleichzeitig ein geschicktes Kanalisieren von Ängsten und damit zusammenhängender Debatten nach den schrecklichen Anschlägen in Paris am 07. , 08. und 09.01.2015, auf den Bildungsbereich und potentielle Attentäter.

So lässt sich zunächst einmal folgende stillschweigende Argumentation aus den Daten und ihrer Analyse erschließen:

Um einen solchen schrecklichen Anschlag zu verhindern, müssen potentielle TäterInnen früh genug erkannt werden, also noch in der Schule. Potentielle TäterInnen werden, so wird präsupponiert, wahrscheinlich schon als Jugendliche auffällig, und zwar, indem sie sich nicht an die gängigen Konventionen halten, also nicht richtig grüßen, nicht jeden Tag in die Schule kommen oder - was junge Frauen und Mädchen betrifft - nicht schwimmen gehen dürfen/wollen. Sie sind eben "integrationsunwillig". Sowohl neoliberale wie rechts-populistische Argumente sind $\mathrm{zu}$ finden: so wird behauptet, dass unsere Gesellschaft ausgenutzt und bedroht wird; dass diese MigrantInnen nicht "zu uns" passen und auch nichts "leisten". Geldstrafen werden als probate Mittel gegen "Integrationsunwilligkeit" angepriesen.

Der nächste argumentative Schritt lautet dann: Wenn man "integrationsunwillig" ist, dann dürfe man nicht in Österreich verweilen. Damit werden vor allem männliche Jugendliche, die möglicherweise sozialisationsbedingt sexistische Einstellungen besitzen, in einem Fehlschluss 
direkt und kausal der Gruppe potentieller Täter zugeordnet (wobei, das muss betont werden, in unseren Gesellschaften sicherlich nicht nur pubertäre männliche Migranten sexistische Einstellungen besitzen).

Ein solcher argumentativer Fehlschluss - nämlich die Konstruktion eines kausalen Zusammenhangs zwischen "integrationsunwilligen" sexistischen Jugendlichen und potentiellen Attentätern - verhindert nicht nur ein Nachdenken über die vielen anderen Ursachen von Radikalisierung; er verhindert auch ein Nachdenken über konstruktive Möglichkeiten der Integration von Jugendlichen aus anderen Kulturkreisen. Und er stigmatisiert in rechts-populistischer Weise eine spezifische Gruppe, konstruiert rasch einen Sündenbock. Sündenböcke dienen, wie man aus der rezenten europäischen (und insbesondere deutschen wie auch österreichischen) Geschichte nur allzu genau weiß, in einer simplistischen Weise der schnellen und einfachen Schuldzuschreibung für komplexe, unerklärte Phänomene.

Doch auch kritische Öffentlichkeiten im oben definierten Sinn konstituierten und artikulierten sich in der untersuchten Zeitspanne. $\mathrm{Zu}$ ihren Stimmen gehören einige NGOs (SOS Mitmensch), Die Junge Sozialdemokratische Partei Österreichs, der Wiener Bürgermeister Michael Häupl, die Grünen, der Geograph und Migrationssoziologe Heinz Fassmann, und einige BildungsexpertInnen, wie die bekannte NGO "Bildung Grenzenlos". Allerdings kamen letztere wesentlich weniger in der Öffentlichkeit zu Wort: Hier kann man deutlich die Rolle der Medien bei der selektiven Berichterstattung festmachen, da diese KritikerInnen vor allem auf soziale Medien angewiesen waren und nur selten in Printmedien interviewt wurden.

In einem viel beachteten und häufig rekontextualisierten Interview mit dem Migrationsexperten und Vizerektor der Universität Wien, Heinz Fassmann, in den Vorarlberger Nachrichten vom 06.02.2015 mit der Headline "Integration braucht keine Strafen, sie braucht Geduld", betonte dieser unter anderem:

Integration heißt, dass die Teilhabe an der Gesellschaft ermöglicht wird. Das geschieht - daraus ergeben sich die Schwerpunkte der Integrationspolitik - indem Menschen am Arbeitsmarkt teilhaben können, wenn sie entsprechende Qualifikationen aufweisen. Daher ist Bildung eine wesentliche Angelegenheit, wie auch die Anerkennungsprozesse von aus dem Ausland mitgebrachten Qualifikationen. Integration bedeutet also nicht Angleichung der Verhaltensweisen oder äußerer Erscheinungsformen.

(Heinz Fassmann [Interview], Vorarlberger Nachrichten 2015)

Bisher ist abgesehen von vereinzelten publizierten Stellungnahmen kein sachlicher Austausch der Politik mit diesen kritischen Öffentlichkeiten in wahrnehmbarer Weise (außer in polarisierten und polarisierenden Fernsehdebatten) erfolgt.

Zusammenfassend lässt sich also aufgrund dieser Begriffsgeschichte und der dekonstruierten Argumentation schließen, dass anstelle von Schuldzuweisungen und argumentativen Fehlschlüssen die komplexen Zusammenhänge benannt und verschiedene Sachverhalte, die fälschlicherweise verbunden werden, getrennt behandelt werden sollten. Es geht um soziokulturelle Unterschiede einerseits, die besprochen und verstanden werden müssen. Anstelle von "Integrationsunwilligkeit" könnte man beispielsweise von "soziokulturellen Unterschieden" sprechen. Andererseits geht es darum, Ursachen von Radikalisierung (von Jugendlichen aus ganz verschiedenen sozialen Schichten) zu erkennen und auch zu beseitigen, einer perspektivenlosen Jugend Alternativen anzubieten. Dies ist aber ein ganz anderes Thema! 


\section{Literatur}

Angouri, Jo/Wodak, Ruth (2014) "'They became big in the shadow of the crisis': The Greek success story and the rise of the far right". Discourse \& Society 25/4: 540-565.

Baker, Paul et al. (2008): "A methodological synergy. CDA and Corpus Linguistics: Analysing Racist Discourses". Discourse \& Society 19/3: 273-306.

Bauman, Zygmunt (1995): "Making and Unmaking of Strangers". Thesis Eleven 43/1: 1-16.

Godin, Benoit (2004): "The New Economy: what the concept owes to the OECD". Research Policy 33: 679-690.

Godin, Benoit (2006): "The knowledge-based economy: conceptual framework or buzzword?" Journal of Technology Transfer 31: 17-30.

Habermas, Jürgen (1998) "Aus der Katastrophe lernen? Ein zeitdiagnostischer Rückblick auf das kurze 20. Jahrhundert". In: Habermas, Jürgen (Hrsg.): Die postnationale Konstellation. Frankfurt a. M.: Suhrkamp.

Jessop, Robert (2008): "The cultural political economy of the knowledge-based economy and its implications for higher education". In: Jessop, Robert/Fairclough, Norman/Wodak, Ruth (eds.): Knowledge Based Economy and Higher Education in Europe. Rotterdam, Sense Publishers: 1-33.

Jessop, Robert/Fairclough, Norman/Wodak, Ruth (eds.) (2008): Knowledge Based Economy and Higher Education in Europe. Rotterdam: Sense Publishers.

Kienpointner, Manfred (1992): Alltagslogik. Stuttgart: Fromann \& Holzboog.

Koller, Veronika/Wodak, Ruth (2008) "Shifting boundaries and emergent public spheres". In: Wodak, Ruth/Koller, Veronika (eds.) (2008): Handbook of Communication in the Public Sphere. Berlin, de Gruyter: 1-17.

Kolvraa, Christoph (i. E.): "The Discourse Theory of Eresto Laclau". In: Wodak, Ruth/Forchtner, Bernhard (eds.): The Handbook of Language and Politics. London: Routledge (in Druck).

Krzyżanowski, Michal/Wodak, Ruth (2009): Politics of Exclusion: Debating Migration in Austria. New Brunswick: Transaction Press.

Laclau, Ernesto/Mouffe, Chantal (1985/2001): Hegemony and Socialist Strategy - Towards a Radical Democratic Politics. London: Verso.

Link, Jürgen (2014): Versuch über den Normalismus. Berlin: Vandenhoeck \& Ruprecht.

Matouschek, Bernd/Wodak, Ruth/ Januschek, Franz (1995): Notwendige Maßnahmen gegen Fremde? Wien: Passagen.

Mouffe, Chantal (2015): "Das System der Mitte kollabiert". Der Standard, 09.-10.05.2015: 8.

Muntigl, Peter/Weiss, Gilbert/Wodak, Ruth (eds.) (2000): EU Discourses of Un/Employment. Amsterdam: Benjamins.

Orwell, George (1946/2006): Politics and the English Language. Peterborough: Broadview Press.

Pelinka, Anton (2013): "Right-Wing Populism: Concept and Typology". In: Wodak, Ruth et al. (eds.): Right-Wing Populism in Europe. Politics and Discourse. London, Bloomsbury: 3-22.

Reisigl, Martin (2014) "Argumentation Analysis and the Discourse-Historical Approach: A Methodological Framework". In: Hart, Chris/Cap, Piotr (eds.) (2014): Contemporary Critical Discourse Studies. London, Bloomsbury: 67-96. 
Reisigl, Martin/Wodak, Ruth (2000): "'Austria First'. A discourse-historical analysis of the Austrian 'anti-foreigner-petition' in 1992 and 1993". In: Reisigl, Martin/Wodak, Ruth (eds.) Semiotics of Racism. Approaches in Critical Discourse Analysis. Vienna, Passagen Verlag: 269-304.

Reisigl, Martin/Wodak, Ruth (2001): Discourse and Discrimination. Rhetorics of Racism and Antisemitism. London: Routledge.

Reisigl, Martin/Wodak, Ruth (2009): "The Discourse-historical Approach (DHA)". In: Wodak, Ruth/Meyer, Michael (eds.) (2009): Methods of Critical Discourse Analysis. London, Sage: 87-121.

Triandafyllidou, Anna/Wodak, Ruth/Krzyżanowski, Michal (eds.): European Media and the European Public Sphere. Basingstoke: Palgrave.

Wengeler, Martin (2003a): Topos und Diskurs. Begründung einer argumentationsanalytischen Methode und ihrer Anwendung auf den Migrationsdiskurs (1960-1985). Tübingen: Niemeyer.

Wengeler, Martin (2003b): "Argumentationstopos als sprachwissenschaftlicher Gegenstand. Für eine Erweiterung linguistischer Methoden bei der Analyse öffentlicher Diskurse". In: Geideck, Susanne/Liebert, Wolf-Andreas (Hrsg.): Sinnformeln. Linguistische und soziologische Analysen von Leitbildern, Metaphern und anderen kollektiven Orientierungsmustern. Berlin/New York, de Gruyter: 59-82.

Wodak, Ruth (2000): "From Conflict to Consensus? The co-construction of a policy paper". In: Muntigl, Peter/Weiss, Gilbert/Wodak, Ruth (eds.): EU Discourses on Un/Employment. Amsterdam, Benjamins: 73-114.

Wodak, Ruth (2011): The Discourse of Politics in Action. Politics as Usual. 2., überarbeitete Auflage. Basingstoke: Palgrave.

Wodak, Ruth (2013a): "'Anything goes!' The Haiderization of Europe". In: Wodak, Ruth et al. (eds.): Right-Wing Populism in Europe. Politics and Discourse. London, Bloomsbury: 2338.

Wodak, Ruth (2013b): "The strategy of discursive provocation - a discourse-historical analysis of the FPÖ's discriminatory rhetoric". In: Feldman, Matthew/Jackson, Paul (eds.): Doublespeak: The Rhetoric of the Far-Rights since 1945. Frankfurt a. M., Ibidem: 101-122.

Wodak, Ruth (2014a): "Argumentation, Political". In: Mazzoleni, Giorgio (eds.): The International Encyclopedia of Political Communication. NY, Wiley \& Sons: i. E..

Wodak, Ruth (2014b): "It would be dangerous to regard European Populism as a triumph of style over substance". http://blogs.lse.ac.uk/europpblog/2014/07/23/it-would-bedangerous-to-regard-modern-european-populism-as-devoid-of-serious-content-or-as-atriumph-of-style-over-substance/ [14.09.2015].

Wodak, Ruth (2015): The Politics of Fear: What Right-Wing Populist Discourses Mean. London: Sage.

Wodak, Ruth/Fairclough, Norman (2010): "Recontextualizing European higher education policies: the cases of Austria and Romania". Critical Discourse Studies 7/1: 19-40.

Wodak, Ruth/Boukala, Salomi (2014a): "Talking about Solidarity and Security in the Age of Crisis: The Revival of Nationalism and Protectionism in the European Union - a Discourse-Historical Approach". In: Carta, Caterina/Morin, Jean-Frederic (eds.): EU Foreign 
Policy through the Lens of Discourse Analysis. Making Sense of Diversity. Farnham, Ashgate: $171-190$.

Wodak, Ruth/Boukala, Salomi (2015): "European Identities and the Revival of Nationalism in the European Union - a Discourse-Historical Approach". Journal of Language \& Politics 14/1: 87-109.

Wodak, Ruth/KhosraviNik, Majid/Mral, Brigitte (eds.) (2013): Rightwing Populism in Europe: Politics and Discourse. London: Bloomsbury.

Wodak, Ruth/Meyer, Michael. (eds.) (2009): Methods of Critical Discourse Analysis. $2^{\text {nd }}$ ed. London: Sage. 